\title{
Mean platelet volume-to-platelet count ratio after elective cardiac surgical procedures is superior in reflecting platelets metabolic hyperactivity compared to other routine morphological platelet indices: A preliminary report
}

Bartłomiej Perek ${ }^{1}$, Piotr Rzymski ${ }^{2,3}$, Anna Komosa ${ }^{4}$, Anna Olasińska-Wiśniewska ${ }^{1}$, Mateusz Puślecki ${ }^{1,5}$, Marek Jemielity ${ }^{1}$, Maciej Lesiak ${ }^{4}$, Sleiman Sebastian Aboul-Hassan ${ }^{6,7}$, Tomasz Stankowski ${ }^{8}$, Zhengyuan Xia ${ }^{9}$, Konrad Stelmark ${ }^{1}$, Jolanta Siller-Matula ${ }^{10}$, Barbara Poniedzialek ${ }^{2}$

${ }^{1}$ Department of Cardiac Surgery and Transplantology, Poznan University of Medical Sciences, Poznan, Poland; ${ }^{2}$ Department of Environmental Medicine, Poznan University of Medical Sciences, Poznan, Poland; ${ }^{3}$ Integrated Science Association (ISA), Universal Scientific Education and Research Network (USERN), Poznan, Poland; ${ }^{4} 1^{\text {st }}$ Department of Cardiology, Poznan University of Medical Sciences, Poznan, Poland; ${ }^{5}$ Department of Medical Rescue, Poznan University of Medical Sciences, Poznan, Poland; ${ }^{6}$ Department of Cardiac Surgery, Medinet Heart Center Ltd., Nowa Sol, Poland; ${ }^{7}$ Department of Cardiac Surgery and Interventional Cardiology, Faculty of Medicine and Medical Sciences, University of Zielona Gora, Poland; ${ }^{8}$ Department of Cardiac Surgery, Sana-Herzzentrum, Cottbus, Germany; ${ }^{9}$ Shenzhen Institute of Research and Innovation, The University of Hong Kong, China; ${ }^{10}$ Department of Internal Medicine II, Division of Cardiology, Medical University of Vienna, Austria

\begin{abstract}
Background: Excessive metabolic excitation of platelets after cardiac procedures may be related to some adverse events but assessment of their metabolic activity is not routine. The purpose of this study was to evaluate which of the basic platelet morphological parameters best reflects their metabolic status. Methods: The blood samples of 22 cardiac surgical patients (mean age of $62.3 \pm 10.3$ years) were taken before surgery (BS), and 1, 24 and 48 hours after the operation. Correlations between morphological platelet parameters (platelet count [PLT], mean platelet volume [MPV], platelet distribution width [PDW] and MPV/PLT) and their metabolic activity (total concentration of malondialdehyde [MDA] and $M D A / P L T)$ were estimated.

Results: Significant decline in PLT after operation (from $223 \pm 44 \times 10^{12} / L$ to $166 \pm 57 \times 10^{12} / L$ ) was accompanied by marked increase in MPV (from $8.4 \pm 0.9 \mathrm{fL}$ to $9.1 \pm 1.2 \mathrm{fL}$ ) and no change of $P D W$. Consequently, MPV/PLT index increased significantly after procedures from (median with IQR) $0.038(0.030-0.043)$ to 0.053 (0.043-0.078). Simultaneously, a significant increase in total platelet $M D A$ content and MDA/PLT was noted reaching peak levels soon after operation. The strongest correlation was observed between MPV/PLT and MDA/PLT $(r=0.56 ; p<0.001)$, although the others were also found to be significant (MDA/PLT vs. MPV; $r=0.35 ; M D A / P L T$ vs. PDW; $r=0.34$ ).

Conclusions: Among basic morphological parameters and indices, the MPV-to-PLT ratio reflects the best metabolic status of platelets in cardiac surgical patients. (Cardiol J)

Key words: cardiac operation, platelet, mean platelet volume, malondialdehyde
\end{abstract}

Address for correspondence: Prof. Bartłomiej Perek, Department of Cardiac Surgery and Transplantology, ul. Długa 1/2, 61-848 Poznań, Poland, tel: +48 618549 233, e-mail: bperek@ump.edu.pl

Received: 16.05.2021 Accepted: 19.10.2021 Early publication date: 15.11.2021

This article is available in open access under Creative Common Attribution-Non-Commercial-No Derivatives 4.0 International (CC BY-NC-ND 4.0) license, allowing to download articles and share them with others as long as they credit the authors and the publisher, but without permission to change them in any way or use them commercially. 


\section{Introduction}

Cardiac surgical operations carried out in the cardio-pulmonary bypass (CBP) are invasive procedures with activation of various cell types and production of numerous biologically active molecules $[1,2]$. Among other blood cells, platelets are involved in developing postoperative systematic inflammatory response syndrome (SIRS) [3]. It was shown previously that SIRS with clinical symptoms was noted in $30 \%$ of all individuals undergoing heart surgeries [4, 5]. Moreover, SIRS occurrence had an adverse effect on the postoperative course and led to higher postoperative morbidity, including organ failure $[4,6]$. On the other hand, the postoperative activation, adherence and aggregation of platelets is mandatory to prevent blood loss at sites of vascular injury and to simultaneously initiate the healing process (a fibrin clot provides a scaffold for attracted inflammatory cells) [7, 8]. Contrary to hyperactivation of platelets, their impaired recruitment on damaged vessels is due to inherited or acquired defects that may promote excessive postoperative bleeding that is usually difficult to counteract by means of surgical maneuvers [9].

So far, many laboratory methods have been developed to test platelet function and metabolic activity that might have potentially applicability after surgical or intravascular procedures $[10,11]$. Some of them, such as flow cytometry or light transmission platelet aggregation, are performed predominantly for basic research because they are time-consuming and require sophisticated analytic equipment [10]. Among rapid tests, a whole blood aggregometer (e.g., Multiplate Analyzer) based on impedance platelet aggregometry enables on-site evaluation of platelet function, but appropriate management with blood samples and analysis of its findings needs experienced laboratory personnel that usually are not available 24 hours a day. Moreover, interpretation of results when platelet count (PLT) is below $120 \times 10^{12} / \mathrm{L}$ may be biased [12]. Of note, not uncommonly, PLT is relatively low after cardiac surgical interventions. Additionally, serum thromboxane $B_{2}$, a stable metabolite of thromboxane $\mathrm{A}_{2}\left(\mathrm{TXA}_{2}\right.$ ) produced by activated platelets, has also been shown as a good marker of platelet's reactivity. However, its measurement is laborious and time-consuming, also when using commercially available colorimetric assays. On the other hand, malondialdehyde (MDA), a broadly and easily assayed marker of lipid peroxidation and oxidative stress [13], is also known to be produced by the platelet's thromboxane synthase in amounts that are equimolar to $\mathrm{TXA}_{2}$ [14]. Therefore, the aforementioned methods may not be considered as routine studies done in all patients in the early postoperative period. In particular, in view of a critical clinical setting that some of them can be related to the abnormal (hyper- or hypo-) activity of platelets, intensivists together with cardiac surgeons must undertake immediate actions to rescue patients. Thus, we need cost-effective and easy-to-perform tests/methods to define platelet metabolic status. It was previously showed that some information withdrawn from basic blood morphology analysis could be clinically relevant [15]. Mean platelet volume (MPV) and platelet distribution width (PDW) were observed to be increased during platelet activation [15]. The others, MPV-to-PLT ratio even better than MPV alone predicted long-term adverse outcomes in patients with ST-segment elevation myocardial infarction (STEMI) undergoing percutaneous coronary interventions (PCIs) [16].

The purpose of this study was to estimate which of the routinely examined basic morphological parameters or their derivatives correlate best with platelet MDA content, a marker of platelet metabolic activity and oxidative stress, after elective standard cardiac surgical procedures carried out from median sternotomy.

\section{Methods}

\section{Patients}

The study involved 22 consecutive patients with a mean age of $62.3 \pm 10.3$ years who underwent elective cardiac surgical procedures from full median sternotomy and in CPB during 1 month period in a single center. The patients who were operated out on a beating heart (off-pump coronary artery bypass grafting [OPCAB]), urgently or emergently, who had any previous cardiac surgical procedures or had to be treated surgically without cessation of antiplatelet (other than acetylsalicylic acid [ASA]) agents as well as on any anticoagulants (including low-molecular-weight heparin [LMWH]) irrespective of the indications, with proven inherited or acquired platelet dysfunction and/or other coagulopathy, PLT less than $120 \times 10^{12} / \mathrm{L}$ on admission and those who had excessive postoperative bleeding followed by chest re-exploration and/or blood products transfusions $(n=2)$ were excluded from the study (Fig. 1). The selected demographic and preoperative clinical data are outlined in Table 1.

All patients expressed written informed consent to participate in this trial. The study protocol 


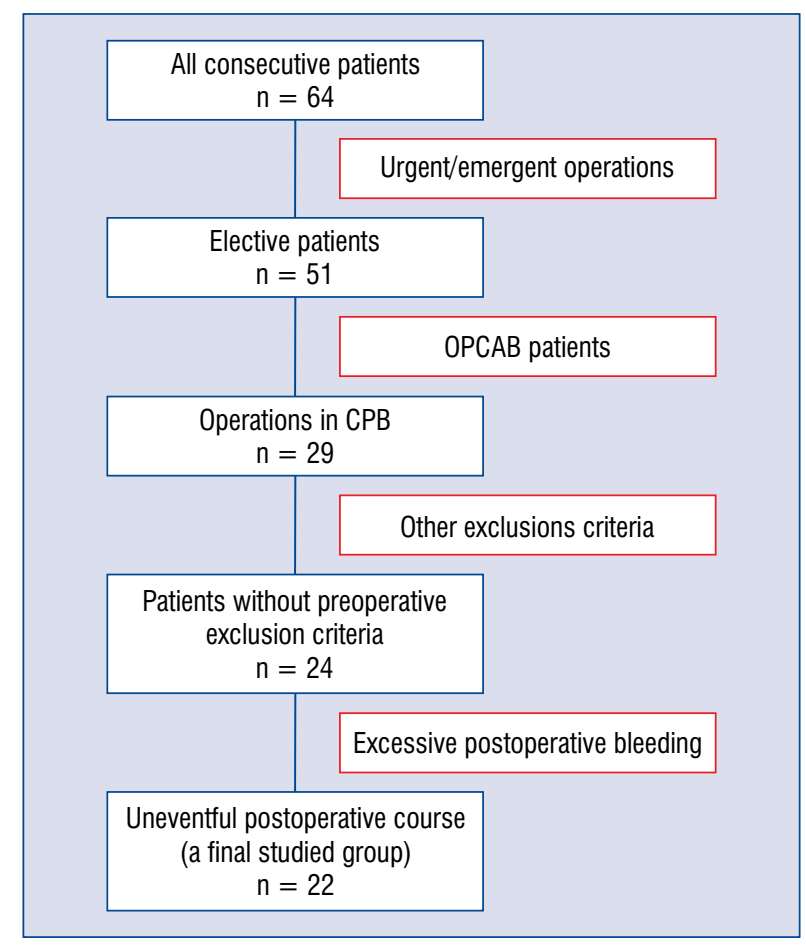

Figure 1. Recruitment process of the consecutive patients. Inclusion and exclusion criteria are indicated in the details contained in the 'Methods' section.

Table 1. Selected preoperative demographic and clinical data of the studied cardiac surgical patients $(\mathrm{n}=22)$

\begin{tabular}{|c|c|}
\hline Parameters* & $N=22$ \\
\hline Gender (male/female) & $16 / 6$ \\
\hline Height $[\mathrm{m}]$ & $1.68 \pm 0.10$ \\
\hline Weight [kg] & $82.9 \pm 14.5$ \\
\hline BMI $\left[\mathrm{kg} / \mathrm{m}^{2}\right]$ & $29.0 \pm 4.1$ \\
\hline Obesity [BMI > $\left.30 \mathrm{~kg} / \mathrm{m}^{2}\right]$ & $9(40.9)$ \\
\hline Hyperlipidemia & $16(72.7)$ \\
\hline Diabetes mellitus & $6(27.3)$ \\
\hline Arterial systemic hypertension & $17(77.3)$ \\
\hline Peripheral artery disease & $5(22.7)$ \\
\hline CVA/TIA & $2(9.1)$ \\
\hline$C K D * * 3+$ & $2(9.1)$ \\
\hline COPD & $4(18.2)$ \\
\hline ACS in history & $7(31.8)$ \\
\hline Previous PCl & $6(27.3)$ \\
\hline \multicolumn{2}{|c|}{$\begin{array}{l}{ }^{*} \text { Continuous variables are expressed as the means } \pm \text { standard } \\
\text { deviation whereas categorical as numbers and percentages; } \\
{ }^{* *} \text { Defined if estimated glomerular filtration rate (eGFR) was below } \\
60 \mathrm{~mL} / \mathrm{min} / 1.73 \mathrm{~m}^{2} \text { following calculation by means of Modification of } \\
\text { Diet in Renal Disease } 4 \text { trial equation (eGFR }=186.3 \times \mathrm{S}_{\mathrm{cr}}{ }^{-1.154} \times \text { age } \\
0.0203 \times 0.742 \text { (if female) } \times 1.21 \text { [if African American]). ACS }- \text { acute } \\
\text { coronary syndrome; BMl - body mass index; CKD - chronic kid- } \\
\text { ney disease; COPD - chronic obstructive pulmonary disease; CVA } \\
\text { - cerebrovascular accident (stroke); PCl - percutaneous coronary } \\
\text { intervention; } \mathrm{S}_{\mathrm{cr}} \text { - serum creatinine concentration; TIA - transient } \\
\text { ischemic attack }\end{array}$} \\
\hline
\end{tabular}

was approved by the Local Bioethical Committee of the Poznan University of Medical Sciences (No. 918/15).

\section{Surgical procedures}

All study participants were operated out from a full median sternotomy. Every patient, before any surgical manipulation on the heart and big vessels received an intravenous injection of heparin in a dose of $300 \mathrm{IU} / \mathrm{kg}$. CPB was conducted through cannulation of the ascending aorta and right atrium. Cold cardioplegic arrest and systemic moderate hypothermia $\left(26\right.$ to $28^{\circ} \mathrm{C}$ ) were applied as myocardial protective methods. At the end of the surgery, protamine sulfate was injected in relation 1:1 to heparin to reverse the latter one action. The anticoagulation effect's accuracy was controlled by activated clotting time measured on-site and in the operating room. The target value of activated clotting time was greater than $400 \mathrm{~s}$.

\section{Perioperative antiplatelet} and anticoagulant management

All but one antiplatelet agent (ASA) was stopped at least 5 days prior to surgery. After operation, the first subcutaneous injections of LMWH in the prophylactic doses were done 6 to 8 hours after surgery (if no excessive bleeding was observed), then twice a day. While postoperative ASA (75 mg) was given in the morning of the next day and then once a day.

\section{Perioperative laboratory examinations}

Sampling points. The blood samples were drawn from a peripheral vein before surgery (on the admission day, usually one date before operation), the second one after patient's transfer from the operative room to the postoperative intensive care unit (post-ICU) (usually within $1 \mathrm{~h}$ after surgery) and then 24 and 48 hours later, irrespective of the patient stay (post-ICU or normal cardiac surgical ward).

Morphological platelets parameters. Besides typical laboratory examinations obligatory for safe monitoring of basic life parameters, including blood morphology and biochemistry, gases (exceptionally taken from artery), samples of $5 \mathrm{~mL}$ of peripheral venous blood were taken for further analysis of platelet metabolic status. Standard morphological parameters of platelets such as PLT, MPV and PDW were entered into an Excel sheet that served as a study database. Eventually, the MPV/PLT ratio was calculated. 


\section{Platelets malondialdehyde content}

Platelet MDA content, one of the major products of lipid peroxidation, was to assess the effect of surgery on the platelet metabolic status. The platelets were collected in the following manner: the platelet-rich plasma was obtained by centrifugation (200 g, $12 \mathrm{~min}$ ), transferred to acid citrate dextrose solution ( $1 / 10 \mathrm{vol}$.) and centrifuged again (900 g, $15 \mathrm{~min})$.

After aspiration of plasma, platelets were suspended in distilled water $(200 \mu \mathrm{L})$. The platelet MDA content was estimated by a commercial TBARS assay kit (Cayman Chemicals, USA) according to the manufacturer protocol and the previous studies [17]. The content was given as $\mu \mathrm{M}$ of MDA. Eventually, MDA content in a single platelet (MDA/PLT) was estimated since invasive surgical procedures can also change PLT significantly.

\section{Data presentation and statistical analysis}

First, continuous variables were tested for normality by means of the Shapiro-Wilk test. If they met the Gaussian distribution assumption, they were presented as the means \pm standard deviation (SD), otherwise as medians and interquartile range (IQR). Time-related changes of all platelet indices were evaluated either by the analysis of variance (ANOVA) with repeated measurements followed, if applicable, by the post hoc Tukey honest significance difference test (for normally distributed continuous variables) or with the use of the Friedman repeated measures ANOVA supplemented by the Dunn multiple rank comparisons (for the other continuous data). Categorical variables were expressed as numbers (n) and percentages (\%). The strength of association between platelet morphological (PLT, MPV, PDW, MPV/PLT) and functional parameters (MDA, MDA/PLT) was tested using the Spearman's correlation coefficient. The latter one was interpreted using the scale provided by Chung and Salkin, where an $r$ between 0.8 and 1.0 (or -0.8 and -1.0 ) was defined as very strong, between 0.6 and 0.8 - as strong, between 0.4 and 0.6 - as moderate, between 0.2 and 0.4 - as weak relationship [18]. A p value $<0.05$ was considered statistically significant. All statistical analyzes were carried out using Statistica 13.3 software (TIBCO Software Inc., Palo Alto, CA, USA).

\section{Results}

\section{Standard platelet indices}

Platelet count. PLT $\left(223 \pm 44 \times 10^{12} / \mathrm{L}\right.$ at baseline [BS]) decreased significantly markedly af- ter surgery $(\mathrm{p}<0.001)$. The post-hoc test revealed significant differences between all postoperative sampling points compared to the BS count. Of note, PLT after reaching the lowest value 24 hours after the operation $\left(166 \pm 57 \times 10^{12} / \mathrm{L}\right)$ started to increase but 48 hours following surgery it was significantly lower $\left(175 \pm 60 \times 10^{12} / \mathrm{L}\right)$ than it was before (Fig. 2A).

Mean platelet volume and platelet distribution width. Although MPV increased markedly after surgery $(\mathrm{p}=0.041)$ but further analysis revealed a significant difference only between $\mathrm{BS}(8.4 \pm 0.9 \mathrm{fL})$ and the last sampling point at $48^{\text {th }}$ hour following operation $(9.1 \pm 1.2 \mathrm{fL} ; \mathrm{p}=0.021$; Fig. $2 \mathrm{~B})$.

Mean PDW values ranges between $54.2 \pm$ $\pm 5.0 \%$ at BS and $57.1 \pm 6.5 \%$ on the $1^{\text {st }}$ day after surgery (Fig. 2C). However, the present calculations failed to show any changes after operations $(\mathrm{p}=0.364)$.

MPV-to-PLT ratio. MPV/PLT index increased significantly after procedures $(\mathrm{p}=0.004)$ and in all postoperative study time points the value of this ratio was markedly higher (peak at the $24^{\text {th }}$ hour following cardiac surgical operations) than it was in the preoperative period (Fig. 2D).

\section{MDA and MDA/PLT}

A significant increase in total MDA platelet content was noted after cardiac surgical interventions $(\mathrm{p}<0.001)$. The highest level (median with IQR) was found soon after the operation (4.3 [2.9-5.2] $\mu \mathrm{M} ; \mathrm{p}<0.001$ vs. BS), then it systematically started to decrease (24 h: 2.6 [2.1-3.2] $\mu \mathrm{M}$; $\mathrm{p}<0.001$ vs. BS), and even $48 \mathrm{~h}$ later (2.2 [1.5-2.9] $\mu \mathrm{M} ; \mathrm{p}=0.027)$ it was still higher than before the procedures (1.0 [0.6-1.5] $\mu \mathrm{M})$.

Since surgery impacted PLT, MDA content in a single thrombocyte (MDA/PLT) was also estimated. Detailed statistical analysis revealed higher medians of MDA/PLT at all sampling points after surgery in comparison to its BS one. The details are shown in Figure 3.

\section{Correlations between platelet morphological and functional parameters}

The strongest association between platelet morphological and markers of metabolic activity were observed between MPV/PLT and MDA/PLT $(\mathrm{r}=0.56 ; \mathrm{p}<0.001)$, although more significant correlations were also found (Fig. 4).

\section{Discussion}

The most crucial finding of this study was the proven association, which was revealed for the 


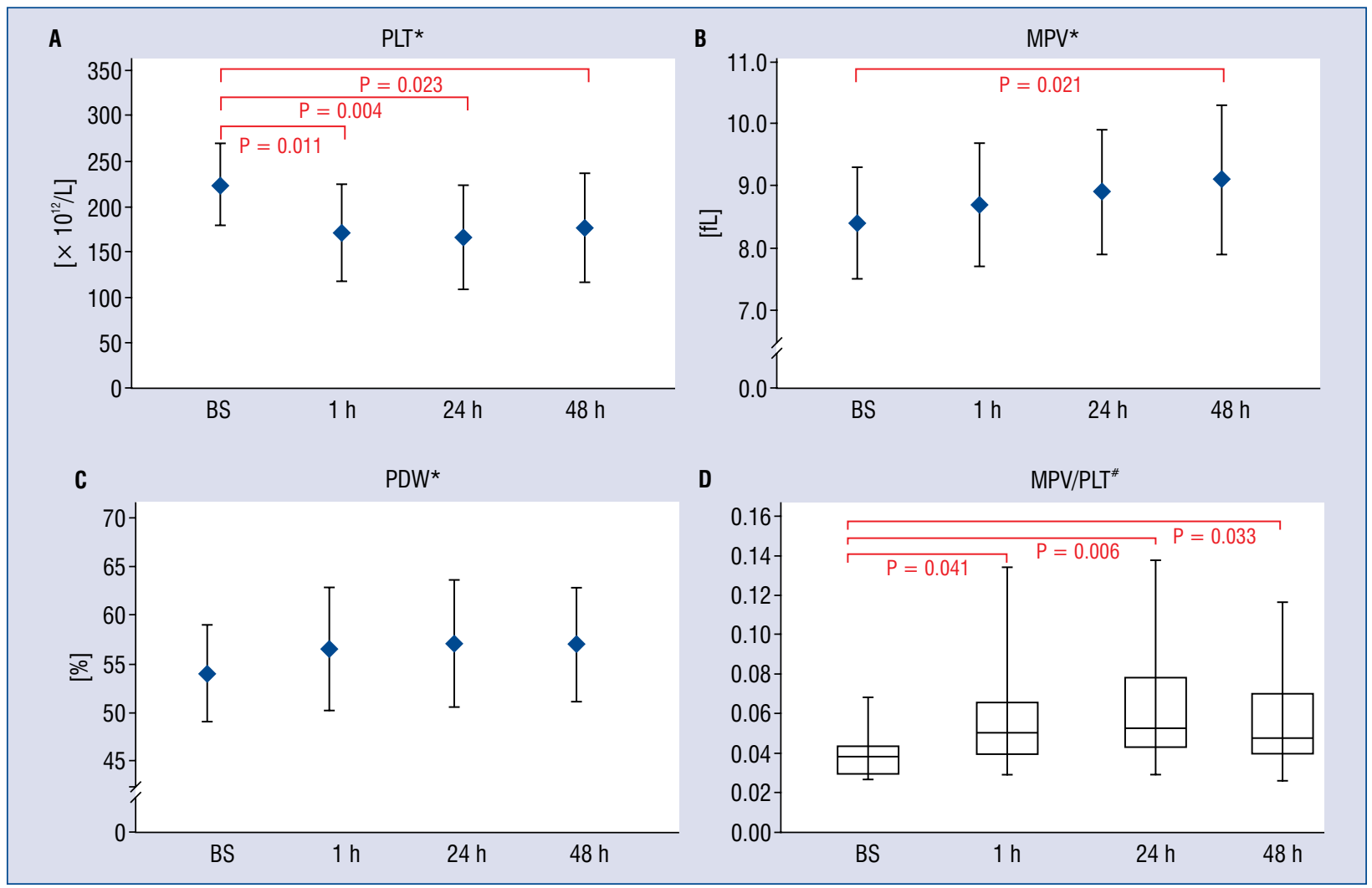

Figure 2. Basic morphological parameters of examined cardiac surgical patients $(n=22)$. Individual points method of presentation (the means with a standard deviation) was applied for parametric data (*; platelet count [PLT; A], mean platelet volume [MPV; B], platelet distribution width [PDW; C]) whereas box plots (the medians with interquartile ranges and total ranges) for nonparametric data (\#; MPV/PLT; D). Only $p$ values of significance in post-hoc tests are on the graphs; BS - baseline.

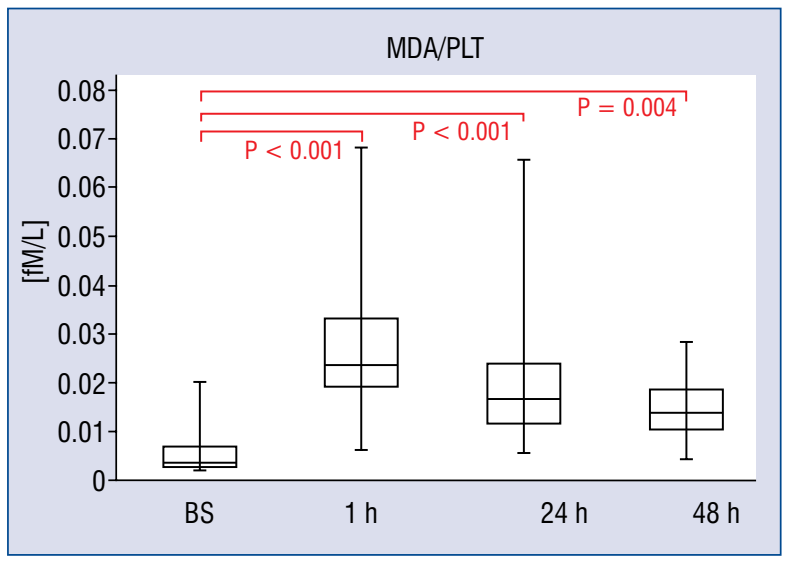

Figure 3. Time-related changes of malondialdehyde (MDA) content in single platelets in the perioperative period in cardiac surgical patients $(n=22)$. MDA/PLT as nonparametric data are presented as the medians with interquartile ranges and total ranges (minimummaximum); BS — baseline; PLT — platelet count. first time according to available research, between metabolic activity of platelets and some basic morphological parameters such as MPV, PDW or MPV/ /PLT that are routinely monitored in the early period after cardiac operations. Therefore, such information about likely metabolic excitation may be easily made available within a short time. The other findings regarding postprocedural changes of morphological parameters, such as temporary decline in PLT as well as increase in MPV are not novel. Up till now, particularly the latter one has been studied extensively (usually together with PDW) in patients with coronary artery disease or after PCI, but not following cardiac operations. Of interest, the easily calculated MPV-to-PLT ratio, in the current study corresponds best with the metabolic activity of platelets, and has aroused scientific interest relatively recently.

Previous reports that PLT usually drops soon after cardiac surgical procedures are supported 


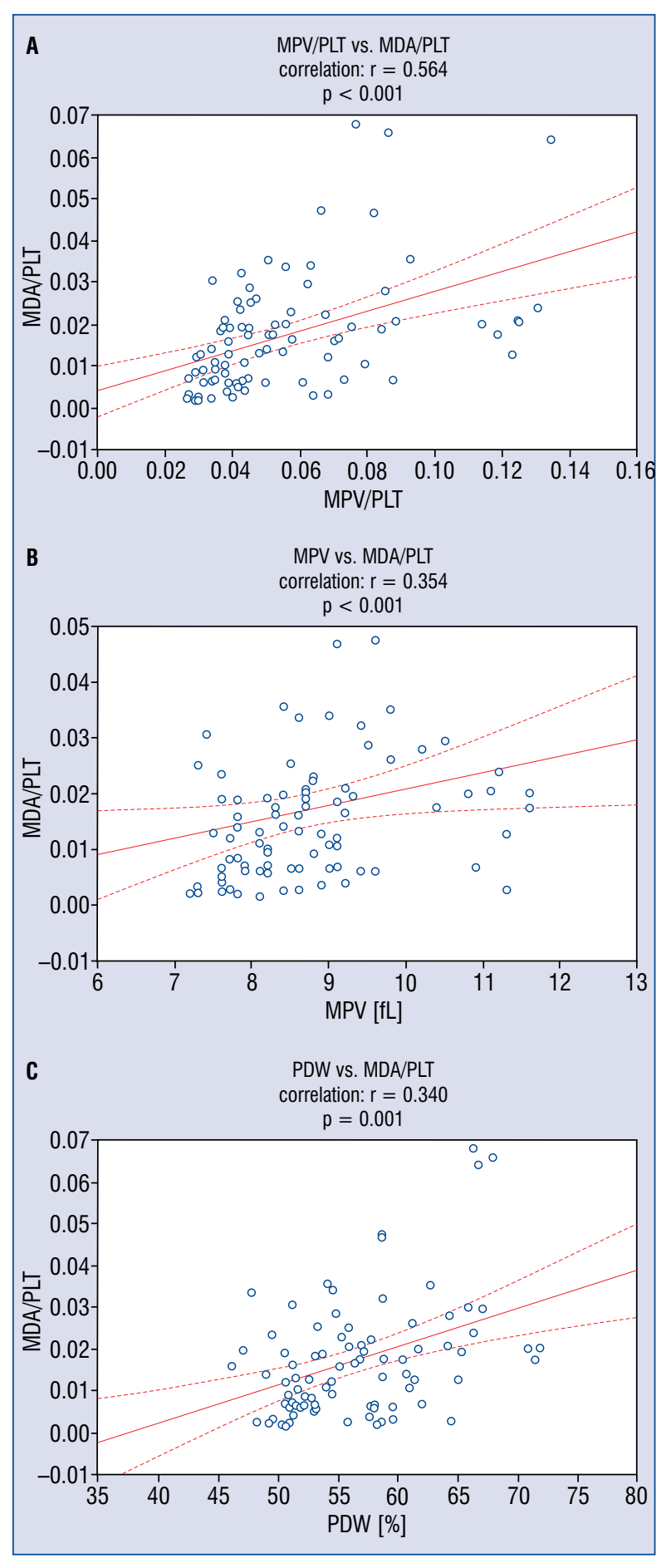

Figure 4. Significant correlation coefficients between platelet morphological parameters and their metabolic activity. Correlations of statistical significance were found between MDA/PLT vs. MPV/PLT (A), vs. MPV (B) as well as vs. PDW (C); MDA - malondialdehyde; MPV - mean platelet volume; PDW - platelet distribution width; PLT — platelet count.

herein, for both adults and in children $[19,20]$. Many factors have been suggested which contrib- ute to low PLT, particularly when CPB is applied this includes; mechanical damage, hemodilution, hypothermia and perioperative treatment with platelet-inhibiting agents [21]. Moreover, blood loss is inevitable during such invasive procedures, especially if more complex procedures with longer CPB time are carried out $[22,23]$. Of note, this effect is usually temporary and not uncommon post 24 hours, at least a partial recovery is usually observed [24]. The latter finding was also confirmed by the present observations. The other basic platelet indices such as MPV and PDW were found to be elevated after procedures associated with iatrogenic injury $[25,26]$. Higher values of MPV and PDW after surgical procedures may be a net result of two biological processes both driven by tissue damage. The trauma-induced mixture of cytokines and reactive oxygen species provokes bone marrow for production and release of platelets to the vascular system due to a differentiation of megakaryocytes [27]. Newly released platelets tend to be larger than mature forms and MPV is often considered a reflection of the average age of these cells. However, the aforementioned reaction of bone marrow in response to surgical trauma may not be harmful. Miceli et al. [26] found that aortic valve replacement in CPB resulted in thrombocytopenia, higher PDW and MPV in the early postoperative period but in the absence of adverse clinical events. On the other side, circulating platelets respond to vessel injury by changes in morphological shape, secretion of granule contents, and aggregation to prevent blood loss. It was previously proved that large size platelets manifested increased enzymatic and metabolic activity as well as prothrombotic potential [28]. Our study supports earlier reports regarding MPV but not PDW. We cannot exclude that recruitment of a larger volume of patients would have revealed more changes of statistical significance.

Monitoring of platelets activity as well as their metabolic status is of importance after medical procedures with extensive iatrogenic tissue damage and SIRS. To assess the metabolic activity of platelets, we have chosen the content analysis of MDA, a product of thromboxane synthase and the marker of lipid peroxidation, oxidative stress and redox imbalance [17]. Oxidative stress altering platelets redox state may lead to their activation and eventually thrombus formation. It was previously observed that increased intraplatelet MDA content was also shown to be related to higher platelet activity, including aggregation [14, 29]. This association was confirmed by the other stud- 
ies that found suppression of platelet MDA levels resulted in inhibition of arachidonate- and collageninduced aggregation [30]. Therefore, pronounced changes in platelet metabolic status may play a role in the functional pathologies responsible for either excessive bleeding or thrombo-embolic adverse events $[4,7]$.

Of note, some basic morphological platelet indices have been suggested to be of clinical prognostic value after both coronary artery bypass grafting (CABG) and acute coronary syndrome patients treated with PCI [31-34]. One of them, MPV was previously found to predict not only the development of saphenous vein graft disease but also mortality and morbidity (e.g., atrial fibrillation) following CABG) [31, 32] Wang et al. [33] showed dynamic changes of MPV during the acute phase of myocardial infarction and higher MPV in patients with high Killip class, suggesting its predictive value in ventricular dysfunction and adverse clinical outcome after angioplasty for acute coronary syndrome. In their study, antiplatelet treatment with pre-used clopidogrel resulted in significant MPV reduction on admission. Not only MPV, but also PDW was found to be associated with noreflow phenomenon after primary PCI, and may be one of the mechanisms responsible for a poor prognosis if the platelet were hyperactive (reflected by rapid elevation of MPV and PDW) [34]. Herein, among platelet indices, the strongest correlation with platelet metabolic activity (MDA) was found for MPV/PLT ratio. The latter one was shown to predict long-term adverse outcomes in patients with STEMI undergoing PCI [16]. In another study, a high MPV/PLT ratio was associated with early vein-graft occlusion and poor postoperative outcomes, including both early and late reduced survival rates [35].

\section{Limitations of the study}

Some limitations of the present study should be stressed. Firstly, the number of patients participating in this clinical study was relatively small. However, even the small group size did enable revealing significant findings. Of course, one cannot exclude the that recruitment of a higher number of individuals would have increased the practical value of the examined parameters. On the other side, the primary stress is put on the findings in the laboratory studies. At least one of them is not routine and requires experienced staff and a well-equipped research laboratory. In the vast majority of studies, such a number is considered sufficient to make the conclusions that follow. There is an awareness that the present findings must be further supported by the clinical outcomes of patients undergoing cardiac surgical procedures. Therefore, it must be stressed that the present research results are treated as groundwork for further prospective clinical studies.

\section{Conclusions}

Heart surgeries as complex and invasive procedures have significant impact on both morphological parameters and indices of their metabolic status. Among basic morphological parameters and indices, the MPV-to-PLT ratio best reflects the metabolic status of platelets in cardiac surgical patients.

\section{Conflict of interest: None declared}

\section{References}

1. Paparella D, Rotunno C, Guida P, et al. Minimally invasive heart valve surgery: influence on coagulation and inflammatory response. Interact Cardiovasc Thorac Surg. 2017; 25(2): 225-232, doi: 10.1093/icvts/ivx090, indexed in Pubmed: 28481998.

2. Hamano K, Kawamura T, Gohra H, et al. Stress caused by minimally invasive cardiac surgery versus conventional cardiac surgery: incidence of systemic inflammatory response syndrome. World J Surg. 2001; 25(2): 117-121, doi: 10.1007/s002680020048, indexed in Pubmed: 11338008.

3. Durandy Y. Minimizing systemic inflammation during cardiopulmonary bypass in the pediatric population. Artif Organs. ndrome2014; 38(1): 11-18, doi: 10.1111/aor.12195, indexed in Pubmed: 24392866.

4. Squiccimarro E, Labriola C, Malvindi PG, et al. Prevalence and clinical impact of systemic inflammatory reaction after cardiac surgery. J Cardiothorac Vasc Anesth. 2019; 33(6): 1682-1690, doi: 10.1053/j.jvca.2019.01.043, indexed in Pubmed: 30772177.

5. Boehne M, Sasse M, Karch A, et al. Systemic inflammatory response syndrome after pediatric congenital heart surgery: Incidence, risk factors, and clinical outcome. J Card Surg. 2017; 32(2): 116-125, doi: 10.1111/jocs.12879, indexed in Pubmed: 27928843.

6. Sasse M, Dziuba F, Jack T, et al. In-line filtration decreases systemic inflammatory response syndrome, renal and hematologic dysfunction in pediatric cardiac intensive care patients. Pediatr Cardiol. 2015; 36(6): 1270-1278, doi: 10.1007/s00246015-1157-x, indexed in Pubmed: 25845941.

7. Ho-Tin-Noé B, Boulaftali Y, Camerer E. Platelets and vascular integrity: how platelets prevent bleeding in inflammation. Blood. 2018; 131(3): 277-288, doi: 10.1182/blood-2017-06-742676, indexed in Pubmed: 29191915.

8. Rodrigues M, Kosaric N, Bonham CA, et al. Wound healing: a cellular perspective. Physiol Rev. 2019; 99(1): 665-706, doi: 10.1152/physrev.00067.2017, indexed in Pubmed: 30475656.

9. Vlot EA, Willemsen LM, Van Dongen EPA, et al. Perioperative point of care platelet function testing and postoperative blood loss in high- 


\section{Cardiology Journal}

risk cardiac surgery patients. Platelets. 2019; 30(8): 982-988, doi: 10.1080/09537104.2018.1542123, indexed in Pubmed: 30411659.

10. Paniccia R, Priora R, Liotta AA, et al. Platelet function tests: a comparative review. Vasc Health Risk Manag. 2015; 11: 133-148, doi: 10.2147/VHRM.S44469, indexed in Pubmed: 25733843.

11. Lordkipanidzé M. Platelet function tests. Semin Thromb Hemost. 2016; 42(3): 258-267, doi: 10.1055/s-0035-1564834, indexed in Pubmed: 26926583.

12. Würtz M, Hvas AM, Christensen KH, et al. Rapid evaluation of platelet function using the Multiplate ${ }^{\circledR}$ Analyzer. Platelets. 2014; 25(8): 628-633, doi: 10.3109/09537104.2013.849804, indexed in Pubmed: 24246241.

13. Komosa A, Rzymski P, Perek B, et al. Platelets redox balance assessment: Current evidence and methodological considerations. Vascul Pharmacol. 2017; 93-95: 6-13, doi: 10.1016/j. vph.2017.06.002, indexed in Pubmed: 28684282.

14. Polzin A, Dannenberg L, Schneider T, et al. Malondialdehyde assay in the evaluation of aspirin antiplatelet effects. Pharmacology. 2019; 103(1-2): 23-29, doi: 10.1159/000493754, indexed in Pubmed: 30355919.

15. Vagdatli E, Gounari E, Lazaridou E, et al. Platelet distribution width: a simple, practical and specific marker of activation of coagulation. Hippokratia. 2010; 14(1): 28-32, indexed in Pubmed: 20411056.

16. Tian C, Song J, He D, et al. Predictive value of mean platelet volume/platelet count for prognosis in acute myocardial infarction. Int Heart J. 2018; 59(2): 286-292, doi: 10.1536/ihj.17-212, indexed in Pubmed: 29563382.

17. Komosa A, Perek B, Rzymski P, et al. Transcatheter aortic valve replacement is associated with less oxidative stress and faster recovery of antioxidant capacity than surgical aortic valve replacement. J Clin Med. 2019; 8(9), doi: 10.3390/jcm8091364, indexed in Pubmed: 31480644.

18. Chung MK. Correlation Coefficient. In: Salkin NJ, ed. Encyclopedia of Measurement and Statistics. Sage Publications, London 2007: 189-201.

19. Karhausen JA, Smeltz AM, Akushevich I, et al. Platelet counts and postoperative stroke after coronary artery bypass grafting surgery. Anesth Analg. 2017; 125(4): 1129-1139, doi: 10.1213/ ANE.0000000000002187, indexed in Pubmed: 28632537.

20. Romlin BS, Söderlund F, Wåhlander H, et al. Platelet count and function in paediatric cardiac surgery: a prospective observational study. Br J Anaesth. 2014; 113(5): 847-854, doi: 10.1093/ bja/aeu194, indexed in Pubmed: 25012582.

21. Squiccimarro E, Jiritano F, Serraino GF, et al. Quantitative and qualitative platelet derangements in cardiac surgery and extracorporeal life support. J Clin Med. 2021; 10(4), doi: 10.3390/ jcm10040615, indexed in Pubmed: 33561947.

22. Ichikawa J, Osada Y, Kodaka M, et al. Association Between Platelet Count and Postoperative Blood Loss in Patients Undergoing Cardiac Surgery With Cardiopulmonary Bypass and Fresh Frozen Plasma Administration Guided by Thromboelastometry. Circ J. 2018; 82(3): 677-683, doi: 10.1253/circj.CJ-17-0712, indexed in Pubmed: 29238008.

23. Lopes CT, Dos Santos TR, Brunori EH, et al. Excessive bleeding predictors after cardiac surgery in adults: integrative review.
J Clin Nurs. 2015; 24(21-22): 3046-3062, doi: 10.1111/jocn.12936, indexed in Pubmed: 26249656.

24. Van Poucke S, Stevens K, Wetzels R, et al. Early platelet recovery following cardiac surgery with cardiopulmonary bypass. Platelets. 2016; 27(8): 751-757, doi: 10.3109/09537104.2016.1173665, indexed in Pubmed: 27164510.

25. Haponiuk I, Chojnicki M, Jaworski R, et al. Inflammatory marker levels after hybrid treatment of selected congenital heart disease in children. Kardiol Pol. 2014; 72(9): 798-805, doi: 10.5603/ KP.a2014.0091, indexed in Pubmed: 24846359.

26. Miceli A, Gilmanov D, Murzi M, et al. Evaluation of platelet count after isolated biological aortic valve replacement with Freedom Solo bioprosthesis. Eur J Cardiothorac Surg. 2012; 41(1): 69-73, doi: 10.1016/j.ejcts.2011.04.015, indexed in Pubmed: 21664141.

27. Chen S, Su Y, Wang J. ROS-mediated platelet generation: a microenvironment-dependent manner for megakaryocyte proliferation, differentiation, and maturation. Cell Death Disease. 2013; 4(7): e722-e722, doi: 10.1038/cddis.2013.253.

28. Gasparyan AY, Ayvazyan L, Mikhailidis DP, et al. Mean platelet volume: a link between thrombosis and inflammation? Curr Pharm Des. 2011; 17(1): 47-58, doi: 10.2174/138161211795049804, indexed in Pubmed: 21247392.

29. Hecker M, Haurand M, Ullrich V, et al. Products, kinetics, and substrate specificity of homogeneous thromboxane synthase from human platelets: development of a novel enzyme assay. Arch Biochem Biophys. 1987; 254(1): 124-135, doi: 10.1016/0003-9861(87)90088-9, indexed in Pubmed: 3579292.

30. Hovinga JK, Felix R, Furlan M, et al. Malondialdehyde formation by blood platelets: a diagnostic test to assess acetylsalicylic acid induced thrombocytopathy? Thromb Res. 1990; 59(1): 89-95, doi: 10.1016/0049-3848(90)90274-g, indexed in Pubmed: 2399531.

31. Kaya U, Koza Y. Predictive value of mean platelet volume in saphenous vein graft disease. Braz J Cardiovasc Surg. 2018 33(4): 317-322, doi: 10.21470/1678-9741-2017-0247, indexed in Pubmed: 30184027.

32. Pala AA, Urcun YS. Is the mean platelet volume a predictive factor for atrial fibrillation developing after coronary artery bypass grafting in elderly patients? Heart Surg Forum. 2020 23(6): E809-E814, doi: 10.1532/hsf.3201, indexed in Pubmed: 33234211.

33. Wang XY, Yu HY, Zhang YY, et al. Serial changes of mean platelet volume in relation to Killip Class in patients with acute myocardial infarction and primary percutaneous coronary intervention. Thromb Res. 2015; 135(4): 652-658, doi: 10.1016/j.thromres.2015.01.033, indexed in Pubmed: 25682433.

34. Cakici M, Cetin M, Balli M, et al. Predictors of thrombus burden and no-reflow of infarct-related artery in patients with ST-segment elevation myocardial infarction: importance of platelet indices. Blood Coagul Fibrinolysis. 2014; 25(7): 709-715, doi: 10.1097/MBC.0000000000000130, indexed in Pubmed: 24806326.

35. Tüysüz ME, Dedemoğlu M. High mean platelet volume to platelet count ratio as a predictor on poor outcomes after CABG. Gen Thorac Cardiovasc Surg. 2020; 68(5): 459-466, doi: 10.1007/ s11748-019-01202-7, indexed in Pubmed: 31515731. 